\title{
Gender Differences in Local and Systemic Reactions to Inactivated Influenza Vaccine, Established by a Meta-Analysis of Fourteen Independent Studies
}

\author{
W.E.P. Beyer ${ }^{1}$, A.M. Palache ${ }^{1}$, R. Kerstens ${ }^{2}$, N. Masurel $^{1 *}$
}

\begin{abstract}
In order to determine whether there is a difference between genders in reported adverse reactions to inactivated influenza vaccine, a computerized database of serological studies was investigated. A standardized questionnaire was used to evaluate vaccine reactogenicity. A total of 1,800 vaccinees in 14 studies were analyzed separately for two age groups $(<60$ and $\geq 60$ years of age). Females reported significantly more local reactions than males. The pooled odds ratio for the outcome measure "any local reaction" was 0.32 (95\% confidence interval, $0.26-0.40$, significant) and $0.54(95 \% \mathrm{Cl}, 0.41-0.70$, significant) for young and elderly adults, respectively. Similar results were obtained for the outcome measure "any systemic reaction." Previous exposure to influenza or influenza vaccine had no influence on reactogenicity. There were no gender differences in seroresponses. In conclusion, gender should be regarded as a predictor of reported reactions to influenza vaccine in both young and elderly adults and should be addressed in future study designs.
\end{abstract}

Possible differences between drug action in men and women have recently caused growing concern in clinical and pharmacological research. In 1993 the U.S. Food and Drug Administration issued new guidelines to stimulate the inclusion of more women in clinical trials and to analyze clinical data by sex (1).

The nature of reactions and side-effects after inactivated whole-virus, split, or subunit influenza vaccines is generally mild and transitory (2). Even if a difference in reactogenicity between the sexes existed, it would have little clinical meaning. In scientific research, however, a difference between the genders, if not addressed, could be a relevant source of error when comparing reactogenicity of different vaccine types, dosages, or administration regimens.

Some earlier papers suggested that the incidence of local or systemic reactions after influenza vac-

\footnotetext{
1 WHÒ National Influenza Centre, Department of Virology, Erasmus University Rotterdam, PO Box 1738, 3000 DR Rotterdam, The Netherlands.

2 Department of Statistics and Data Management, Solvay Duphar bv, PO Box 900, 1380 DA Weesp, The Netherlands.
}

cination was higher in adult females than in adult males (3-7). However, two of these studies $(3,5)$ used the influenza A/New Jersey/76 (H1N1) swine strain, which showed unusual characteristics of reactogenicity. In two other reports $(4,7)$, data on gender differences were presented as an accidental finding, and one investigation (6) had only small study groups.

In an attempt to substantiate a possible difference between genders in vaccine reactogenicity, we analyzed computerized data on reported reactions from trials with an influenza subunit vaccine performed during a ten-year period. In these trials the same standardized questionnaire to evaluate reactions had been used. Data on prevaccination status (vaccinations in previous years, and prevaccination serology) and seroresponse to the vaccine were also analyzed.

\section{Materials and Methods}

Sources of Data and Study Design. In the period 1982-1991, 30 serological studies with influenza subunit vaccine (Solvay Duphar, The Netherlands) were conducted for registration purposes. All studies were performed according to standard operating procedures to comply with the Dutch Good Clini- 
Table 1: Numbers of subjects for meta-analysis (MA) of reported reactions.

\begin{tabular}{lccccccccc}
\hline $\begin{array}{l}\text { Age } \\
\text { group }\end{array}$ & $\begin{array}{c}\text { MA } \\
\text { group }\end{array}$ & $\begin{array}{c}\text { Study } \\
\text { no. }^{\mathrm{a}}\end{array}$ & $\begin{array}{c}\text { Hema- } \\
\text { glutinin } \\
\text { dose }(\mu \mathrm{g})\end{array}$ & $\begin{array}{c}\text { No. of } \\
\text { subjects }\end{array}$ & $\begin{array}{c}\text { No. of } \\
\text { females }\end{array}$ & $\begin{array}{c}\text { No. of } \\
\text { males }\end{array}$ & $\begin{array}{c}\text { Any } \\
\text { local } \\
\text { symptom }\end{array}$ & $\begin{array}{c}\text { Any } \\
\text { systemic } \\
\text { symptom }\end{array}$ & $\begin{array}{c}\text { Incon- } \\
\text { venience }^{b}\end{array}$ \\
\hline Young & 1 & 1 & 30 & 116 & 66 & 50 & 56 & 26 & 9 \\
& 2 & 4 & 30 & 125 & 24 & 101 & 39 & 15 & 5 \\
& 3 & 7 & 30 & 85 & 44 & 41 & 34 & 20 & 6 \\
4 & 9 & 30 & 101 & 49 & 52 & 27 & 23 & 6 \\
5 & 11 & 30 & 69 & 42 & 27 & 31 & 16 & 4 \\
6 & 11 & 60 & 71 & 43 & 28 & 36 & 18 & 7 \\
7 & 15 & 30 & 122 & 70 & 52 & 35 & 16 & 2 \\
8 & 18 & 30 & 148 & 85 & 63 & 62 & 38 & 3 \\
9 & $19 c$ & 30 & 41 & 19 & 22 & 28 & 5 & 0 \\
10 & 21 & 30 & 70 & 39 & 31 & 31 & 19 & 4 \\
11 & 21 & 60 & 71 & 39 & 31 & 36 & 12 & 5 \\
12 & 22 & 30 & 72 & 30 & 42 & 35 & 15 & 3 \\
13 & 22 & 45 & 72 & 43 & 29 & 45 & 15 & 6 \\
14 & 22 & 60 & 72 & 30 & 42 & 40 & 17 & 3 \\
15 & 24 & 30 & 100 & 32 & 68 & 24 & 14 & 4 \\
16 & 28 & 30 & 99 & 51 & 48 & 43 & 18 & 4 \\
17 & 17 & 30 & 114 & 55 & 59 & 38 & 19 & 7 \\
18 & $19 c$ & 30 & 139 & 71 & 68 & 63 & 40 & 13 \\
19 & 25 & 30 & 113 & 59 & 54 & 42 & 32 & 9 \\
\hline
\end{tabular}

a According to reference 9 .

${ }^{b}$ Number reporting.

c Study 19 was subdivided into two meta-analysis groups, according to subjects' age.

cal Practice guidelines, after obtaining ethics committee approval. One study was a multicentre, randomized, placebocontrolled double-blind study recently published (8); the other studies have not been published. However, an overview of all of these studies has been published (9). Study participants were either young healthy volunteers recruited from medical teaching institutions, subjects of all ages visiting general practices, or residents of nursing homes.

The study procedures were virtually identical for all studies. Briefly, before vaccination, volunteers were interviewed to obtain their history of previous vaccinations and to exclude those with a known allergy to chicken proteins. Suitable subjects were asked to give written informed consent. A prevaccination blood sample was taken, and $0.5 \mathrm{ml}$ of vaccine was then administered intramuscularly. A second blood sample was taken two to four weeks after vaccination. Homologous antibody titres of blood samples were determined by standard hemagglutination-inhibition tests (10-12). Further details on study designs, sizes, vaccine composition, and dosages have been published previously (9).

In the studies in which data on reactogenicity were collected, each volunteer received a standard questionnaire on which to report adverse effects during the $48 \mathrm{~h}$ period following vaccination. Questionnaire forms were distributed on the day of vaccination and were collected when the second blood sample was drawn. The questionnaire addressed seven local and five systemic symptoms, as well as the degree of inconvenience these symptoms caused. Local reactions included redness, swelling, itching, warmth, pain on contact or continuous pain at the site of vaccination, and restriction in arm movement. Systemic reactions included fever, increased sweating, headache, malaise, and insomnia. Inconvenience was recorded as absent, slight, moderate, or severe. Afterwards, two additional summary variables were derived: "any local reaction" and "any systemic reaction."
Selection of Data. Dosages used in the trials varied from 0 to $180 \mu \mathrm{g}$ hemagglutinin (total amount of hemagglutinin for all virus strains). Most trials were single-dose experiments, and some were dose-response studies. Most common was a dose of $30 \mu \mathrm{g}$ hemagglutinin (trivalent vaccine with $10 \mu \mathrm{g}$ hemagglutinin per strain). For the present analysis, a dose range of 30 to $60 \mu \mathrm{g}$ hemagglutinin was chosen because this interval corresponds to the total amount of hemagglutinin in current vaccines (trivalent or tetravalent vaccines with 10 or $15 \mu \mathrm{g}$ hemagglutinin per strain). If a trial included more than one dose, dose groups were analyzed separately. Subjects $<60$ years of age ("young") and subjects $\geq 60$ years of age ("elderly") were analyzed separately. Meta-analysis groups were formed according to trial, dosage, and age.

Statistical Procedures. The data of all groups in the meta-analysis were arranged in $2 * 2$ matrix tables (presence and absence of reaction vs. males and females) and subjected to a homogeneity test between meta-analysis groups according to Breslow and Day (13). If homogeneity could be confirmed, the Cochran-Mantel-Haenzel method of meta-analysis, as modified by Yusuf et al. (14), was applied, resulting in pooled odds ratios and their $95 \%$ confidence intervals (CIs). In the case of no difference between the sexes, the pooled odds ratio value is 1 . A pooled odds ratio less than 1 means that the frequency of a reaction in females is higher than in males, and vice versa. Using the same procedure, reactions versus dosage, previous vaccination, and prevaccination antibody status, and seroresponse variables versus gender were subjected to meta-analysis.

\section{Results}

Definite Number of Subjects for Analysis, and Overall Frequency of Reactions. Of the 30 serolog- 

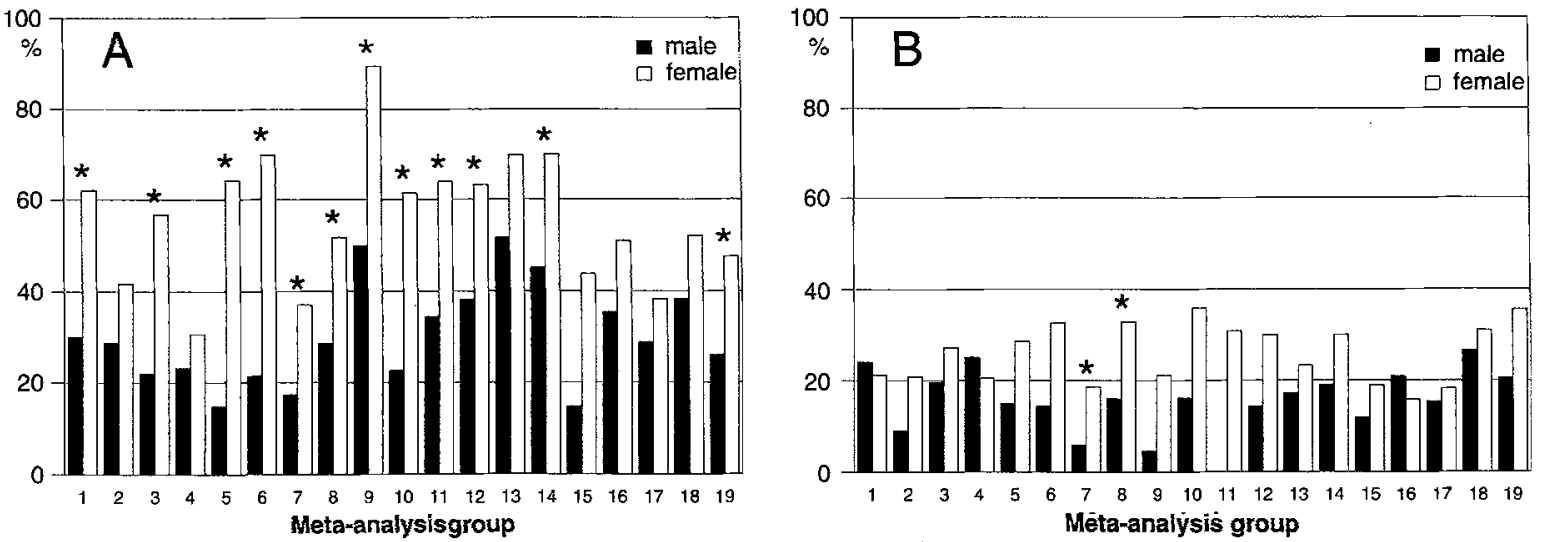

Figure 1: Frequencies of reported vaccine reactions, according to meta-analysis groups and gender. 1A: any local symptom reported; 1B: any systemic symptom reported. * represents significant difference between males and females.

ical studies performed between 1982 and 1991 (9), 15 studies (with 2,267 subjects) included registration of adverse reactions. In one of these studies (with 115 elderly subjects, most of whom suffered from dementia) virtually no reactions were reported; this study was excluded. Of the remaining vaccinees, 352 were vaccinated with doses of less than $30 \mu \mathrm{g}$ or greater than $60 \mu \mathrm{g}$ hemagglutinin, and these were also excluded. Thus, the individual data of 1,800 subjects from 14 studies could be included, consisting of 1,434 young adults (range 16 to 59 years of age, mean 24.9) and 366 elderly (62 to 94 years of age, mean 74.7). Subjects were divided into 19 meta-analysis groups (Table 1 ).

Of the local reactions, pain on contact was reported most frequently; of systemic reactions, headache. Data on inconvenience were included as either moderate or severe; these latter numbers were small in all studies, confirming the generally mild nature of vaccine reactions. There were large differences between the meta-analysis groups in the frequencies of reactions reported: for example, in meta-analysis group $9,68.3 \%$ of the vaccines reported "any local reaction," compared to only $26.7 \%$ in meta-analysis group 4 .

Influence of Gender on Frequency of Reactions. For every single reaction, the frequency and odds ratio per meta-analysis group were calculated. As an example, Figure 1 presents results for "any local reaction" and "any systemic reaction." A significantly higher frequency of "any local reaction" in females than in males was detected in 13 of the 19 meta-analysis groups. For "any systemic reaction," only meta-analysis groups 7 and 8 showed a significant difference between the sexes.

The test on homogeneity indicated the presence of homogeneity for all reactions in both age groups (data not shown). Table 2 presents the pooled odds ratios per symptom. In the young adults, a clear difference between genders was present for each of the local reactions: all seven pooled odds ratio values were less than 1 , reaching a significant level in six groups. The pooled odds ratio for "any local reaction" was also significant: 0.32 (95\% CI: 0.26-0.40). A gender difference for systemic reactions was present, although less pronounced. Pooled odds ratio values were generally greater for systemic than for local reactions but were still significantly less than 1 for "sweating," "headache," and "any systemic reaction"; the symptom "fever" may be a borderline case. Two systemic reactions, "malaise" and "insomnia," were associated with pooled odds ratio values close to 1 , thus showing no gender difference. Finally, significantly more women suffered from moderate or severe inconvenience.

A gender difference for local reactions was also observed in the elderly. Pooled odds ratio values for all seven reactions and for "any local reaction" were less than 1 . Only the values for "itching" and "warmth" did not reach a significant level. The systemic reactions "sweating" and "malaise" scored lower than in the young adults, whereas "fever" and "headache" scored higher. The score for "moderate or severe inconvenience" was very similar to that of the young age group.

There was one unexpected difference between the age groups: "insomnia" was gender neutral in young adults but was found to be significantly more frequent in elderly males than in females. As a consequence, the summary variable "any systemic reaction" showed a high and nonsignificant pooled odds ratio $(0.67,95 \%$ CI: $0.42-1.08)$ in the elderly. When "any systemic reaction" was recalculated without the symptom "insomnia" (in Ta- 
Table 2: Pooled odd ratios (POR) of gender differences for vaccine reactions in young and elderly vaccinees.

\begin{tabular}{lll}
\hline Vaccine reaction & POR young & POR elderlya \\
\hline Redness & $0.30(0.22-0.43)+$ & $0.29(0.15-0.54)+$ \\
Swelling & $0.32(0.22-0.46)+$ & $0.31(0.17-0.60)+$ \\
Itching & $0.33(0.19-0.57)+$ & $0.69(0.36-1.34)-$ \\
Warmth & $0.31(0.22-0.44)+$ & $0.54(0.28-1.05)-$ \\
Pain on contact & $0.36(0.28-0.45)+$ & $0.43(0.26-0.71)+$ \\
Continuous pain & $0.70(0.43-1.12)-$ & $0.21(0.06-0.83)+$ \\
Restricted arm movement & $0.42(0.28-0.65)+$ & $0.45(0.21-0.96)+$ \\
& & \\
Fever & $0.57(0.32-1.00)-$ & $0.89(0.34-2.33)-$ \\
Increased sweating & $0.55(0.34-0.88)+$ & $0.33(0.14-0.77)+$ \\
Headache & $0.42(0.30-0.59)+$ & $0.70(0.39-1.26)-$ \\
Malaise & $0.97(0.61-1.52)-$ & $0.43(0.21-0.91)+$ \\
Insomnia & $0.92(0.52-1.63)-$ & $2.95(1.29-6.74)+$ \\
Inconvenience ${ }^{b}$ & $0.36(0.22-0.59)+$ & $0.39(0.18-0.84)+$ \\
Any local reaction & & \\
Any systemic reaction & $0.32(0.26-0.40)+$ & $0.54(0.35-0.82)+$ \\
Any systemic reaction after correctionc & $0.54(0.41-0.70)+$ & $0.67(0.42-1.08)-$ \\
a Pooled odds ratios with 95\% confidence intervals and significance (+, significant; -, not significant; \\
at the 5\% level). & $0.51(0.39-0.67)+$ & \\
b Moderate or severe inconvenience. & &
\end{tabular}

ble 2, "any systemic reaction corrected"), the pooled odds ratio became significant in the elderly and was similar to that of young adults $(0.57$, 95\% CI: 0.35-0.94).

Influence of Dose, Previous Vaccinations, and PreVaccination Antibody Status on Frequency of Reactions. Three studies including 425 subjects (studies 11, 21, and 22; Table 1) allowed a comparison of reactions for doses of 30 and $60 \mu \mathrm{g}$ hemagglutinin by a similar procedure of meta-analysis (not shown). There was no dose-response relationship for systemic reactions, but two single local reactions (swelling, continuous pain) were reported more frequently in vaccinees who received the higher dosage.

Data were analyzed for the influence of vaccinations against influenza in previous years. For all reactions, no differences were detected between subjects previously vaccinated and those not previously vaccinated (data not shown). Reaction frequencies were analyzed according to the prevaccination antibody status (seronegative vs. seropositive, and not protected vs. protected, prior to vaccination), separately for the three virus (sub)types. Prevaccination status had no influence on reactogenicity in any case (data not shown).

Influence of Gender on Serological Variables. Quantitative and dichotomous serological variables (geometric mean titres, and percentages of subjects exceeding protective threshold titres, prior to and after vaccination) were calculated according to gender (data not shown) and were found to be similar for males and females. There was no relationship between gender and seroresponse in our data.

\section{Discussion}

Despite the use of a standard questionnaire, trials differed considerably in the frequency of reported reactions (Table 1). These inter-trial differences were probably due to differences in the attitude of researchers and vaccinees. Our trials were conducted in different study centres and by different researchers, and vaccinees differed in their educational and social background. Some studies reporting very high reactogenicity were conducted in medical students who were obviously highly motivated to observe even the slightest symptom. On the other hand, trials in the elderly may easily underreport reactions due to dementia and forgetfulness in the subjects. The criterion "age" may, therefore, measure not only immunological differences due to biological age but also psychological, mental, and medical differences. Therefore, the two age groups were analyzed separately. It could thus be shown here that there were no essential differences between age groups.

It is well known that those persons previously unexposed to influenza (sub)types (unprimed sub- 
jects) suffer from more reactions than those previously exposed [for example, young adults vaccinated in 1976 with the A/New Jersey/76 (H1N1) swine strain $(3,5)$, young adults vaccinated in 1977 with the A/USSR/77 (H1N1) strain (15), or young children vaccinated with any influenza subtype (2)]. The populations in the trials presented here consisted of adults, and the trials were performed between 1982 and 1991, i.e. during an interpandemic period, and did not include the NJ/76-strain. It could be assumed that virtually all participants had already been exposed to natural influenza (sub)types A-H3N2, A-H1N1, and $B$. Indeed, a history of previous vaccinations and an antibody-status report required prior to vaccination were found not to influence the frequency of reactions.

Published reports show a dose-response relationship for local reactions and, though less pronounced, for systemic reactions $(6,8,16-19)$. We controlled possible dose bias by dividing studies with different doses into separate groups. We confirmed that a higher dose (60 vs. $30 \mu \mathrm{g}$ hemagglutinin) was associated with a higher frequency of some local reactions.

During the period 1982 to 1991 , the industrial manufacture of the subunit vaccine used was based on the procedure of Bachmayer et al. (20) and had not been modified. The strain composition of the vaccines, however, changed every year (see Table 2 of reference 9). It is conceivable that certain influenza strains could be more reactogenic than others, which may have contributed to the heterogeneity between studies.

After applying a method of meta-analysis, gender differences were established for both local and systemic reactions and for both age classes: female vaccinees reported more reactions than male vaccinees (Table 2). Moreover, a history of previous vaccinations did not influence reported reactions. These two results are in good accordance with a large single vaccination trial by Govaert et al. (7), who applied a similar method of data assessment in a large study group (1,806 participants). The only difference was that they also found a significantly higher frequency of females reporting "any systemic reaction," something we did not observe. This may be due to our inclusion of the symptom "insomnia" in our questionnaire, which was not included by Govaert et al. (7). After correcting for this possibly untypical symptom, we found our results to be similar to theirs.
When interpreting the results of our study, one should keep in mind that data on reactions were not collected by a physician when symptoms occurred but were reported by the vaccinees themselves. The data do not represent actual reactions but the frequency of vaccine reactions reported and may, therefore, be influenced by individual mechanisms such as understatement and exaggeration, i.e. by gender-specific, psychological rather than physiological factors. The concept that vaccine reactions may be mediated by physiological gender differences can be neither denied nor supported by our data. It is, however, remarkable that fever, the most "objective" reaction, was not found for a clear gender difference, again in accordance with the study of Govaert et al. (7). Reports in the field of medical psychology have documented gender differences in the awareness of perception of pain (21-24).

In conclusion, our data suggest that gender is a predictor of reported influenza vaccine reactions in young and elderly adults. We recommend, therefore, that groups with similar numbers of females and males be studied when investigating vaccine reactogenicity, and that gender be included as an independent variable in the statistical analysis.

\section{Acknowledgements}

The authors wish to thank Dr. Marc Sprenger for his valuable comments. The study was financially supported by The Foundation of Respiratory Virology, Notably Influenza (SRVI), Rotterdam, The Netherlands.

\section{References}

1. Merkatz RB, Temple R, Sobel S, Kessler DA, the Working Group on Women in Clinical Trials: Women in clinical trials of new drugs. A change in Food and Drug Administration policy. New England Journal of Medicine 1993, 329: 292-296.

2. Centers for Disease Control: Prevention and control of influenza. Recommendations of the Immunization Practices Advisory Committee (ACIP). Morbidity and Mortality Weekly Report 1991, 40: 1-15.

3. Mostow SR, Eickhoff TC, Chelgren GA, Retailliau HF, Castle M: Studies of inactivated virus vaccines in hospital employees: reactogenicity and absenteeism. Journal of Infectious Diseases 1977, 136, Supplement: 533-538.

4. Masurel N, Laufer J: A one-year study of trivalent influenza vaccines in primed and unprimed volunteers: immu- 
nogenicity, clinical reactions and protection. Journal of Hygiene 1984, 92: 263-276.

5. Cate TR, Kasel JA, Couch RB, Six HR, Knight V: Clinical trials of bivalent A/New Jersey/76 - ANictoria/75 vaccines in the elderly. Journal of Infectious Diseases 1977 , 136, Supplement: 518-525.

6. Cate TR, Couch RB, Parker D, Baxter B: Reactogenicity, immunogenicity, and antibody persistence in adults given inactivated influenza virus vaccines -1978 . Reviews of Infectious Diseases 1983, 5: 737-747.

7. Govaert TME, Dinant GJ, Aretz K, Masurel N, Sprenger MJW, Knottnerus JA: Adverse reactions to influenza vaccine in elderly people: randomized double blind placebo controlled trial. British Medical Journal 1993, 307: 988-990.

8. Palache AM, Beyer WEP, Sprenger MJW, Masurel N, De Jonge $S$, Vardy A, Charpentier B, Noury J, Van Beek WCA, Borst RJA, Ligthart GJ, Keren G, Rubinstein E: Antibody response after influenza immunization with various vaccine doses: a double-blind, placebo-controlled, multi-centre, dose-response study in elderly nursinghome residents and young adults. Vaccine 1993, 11: 3-9.

9. Palache AM: Influenza subunit vaccine - ten years' experience. European Journal of Clinical Research 1992, 3: 117-138.

10. Dowdle WR, Kendal AP, Noble GR: Influenza viruses. In: Lennette EH, Schmidt NJ (ed): Diagnostic procedures for viral, rickettsial and chlamydial infections. American Public Health Association, Washington, DC, 1979, p. 585-609.

11. Masurel N, Ophof P, De Jong P: Antibody response to immunization with influenza A/USSR/77 (H1N1) virus in young individuals primed or unprimed for A/New Jersey/76 (H1N1) virus. Journal of Hygiene 1981, 87: 201-209.

12. Beyer WEP, Masurel N: Antigenic heterogeneity among influenza $A(H 3 N 2)$ field isolates during an outbreak in $1982 / 83$, estimated by methods of numerical taxonomy. Journal of Hygiene 1985, 94: 97-109.

13. Breslow NE, Day NE: The analysis of case-control studies. Statistical methods in cancer research. Volume 1. International Agency of Research on Cancer, Lyon, 1980, p. 142.

14. Yusuf S, Peto R, Lewis J, Collins R, Sleight P: Beta blockade during and after myocardial infarction: an overview of the randomized trials. Progress in Cardiovascular Diseases 1985, 27: 335-371.

15. Nicholson KG, Tyrrell DAJ, Harrison P, Potter CW, Jennings R, Clark A, Schild GC, Wood JM, Yetts R, Seagroatt $V$, Huggins A, Anderson SG: Clinical studies of monovalent inactivated whole virus and subunit A/USSR/77 (H1N1) vaccine: serological responses and clinical reactions. Journal of Biological Standardization 1979, 7: 123-136.

16. Jennings $\mathrm{R}$, Potter $\mathrm{CW}$, Massey PMO, Duerden BI, Martin J, Bevan AM: Responses of volunteers to inactivated influenza virus vaccines. Journal of Hygiene 1981, 86: $1-16$.

17. Jennings R, Smith TL, Mellersh AR, Clark A, Spencer RC, Potter CW: Antibody response and persistence in volunteers following immunization with varying dosages of a trivalent surface antigen influenza virus vaccine. Journal of Hygiene 1985, 94: 87-95.

18. Goodeve A, Potter CW, Clark A, Jennings R, Schild GC, Yetts R: A graded-dose study of inactivated, surface antigen influenza $B$ vaccine in volunteers: reactogenicity, antibody response and protection to challenge virus infection. Journal of Hygiene 1983, 90: 107-115.

19. Beyer WEP, Teunissen MWE, Diepersloot RJA, Masurel $\mathrm{N}$ : Immunogenicity and reactogenicity of two doses of a trivalent influenza split vaccine. An open randomized study in healthy, unprotected, adult volunteers. Journal of Drug Therapy Research 1986, 11: 369-373.

20. Bachmayer $H$, Liehl E, Schmidt G: Preparation and properties of a novel influenza subunit vaccine. Postgraduate Medical Journal 1976, 52: 360-367.

21. Nathanson CA: Illness and the feminine role: a theoretical review. Social Science and Medicine 1975, 9: 57-62.

22. Verbrugge LM: Females and illness: recent trends in sex differences in the United States. Journal of Health and Social Behavior 1976, 17: 387-403.

23. Nathanson CA: Sex, illness, and medical care. A review of data, theory, and method. Social Science and Medicine 1977, 11: 13-25.

24. Waldron I: Sex differences in iflness incidence, prognosis and mortality; issues and evidence. Social Science and Medicine 1983, 17: 1107-1123. 